\title{
Linfomas en pacientes con VIH: una asociación compleja y frecuente. Revisión narrativa de la literatura
}

\section{HIV-related lymphomas: a complex and frequent association. A narrative review of the literature}

\author{
Guillermo Andrés Herrera-Rueda' (iD, Julie Melissa Mogollón-Vargas² iD, \\ Angie Yarlady Serrano-García ${ }^{3}$, Lina María Gaviria-Jaramillo ${ }^{4}$ (iD)
}

\begin{abstract}
Resumen. En Colombia, la infección por el virus de la inmunodeficiencia humana (VIH) es una epidemia en aumento. Ante la mejoría de la supervivencia general con la terapia antirretroviral altamente efectiva, la aparición de neoplasias malignas ha ido desplazando las complicaciones infecciosas como principal problema en esta población. Los linfomas no Hodgkin son las neoplasias malignas definitorias de síndrome de inmunodeficiencia adquirida (SIDA) más frecuentes después del sarcoma de Kaposi, siendo el linfoma B difuso de células grandes y el linfoma de Burkitt las variantes más comunes; por otra parte, el riesgo de desarrollar linfoma de Hodgkin clásico es 5 a 20 veces mayor en pacientes positivos para VIH en comparación con la población general. Realizar un diagnóstico temprano es un reto, debido a infecciones oportunistas y a la presentación atípica de la enfermedad en este grupo de pacientes. El tratamiento de la enfermedad también supone un desafío debido a las comorbilidades y al estado funcional de los pacientes al momento del diagnóstico; sin embargo, en conjunto con esquemas combinados de quimioterapia y el uso concomitante de la terapia antirretroviral, se ha mejorado considerablemente el pronóstico, el cual actualmente se acerca al de los pacientes seronegativos. Por medio de esta revisión, se pretende describir las principales características de los linfomas asociados al VIH, de tal forma que permita a los trabajadores en salud, tener mayores elementos para el abordaje integral de esta población en nuestro país.
\end{abstract}

\footnotetext{
1 Médico, Especialista en Medicina Interna. Fellow en Hematología Clínica, Universidad de Antioquia. Medellín, Colombia. E-mail: guillermo.herrera@udea.edu.co

2 Médica, Especialista en Medicina Interna. Unidad de Hematología, Hospital Universitario de Santander. Bucaramanga, Colombia.

${ }^{3}$ Estudiante de Medicina, Universidad Industrial de Santander. Bucaramanga, Colombia.

${ }^{4}$ Médica, Especialista en Medicina Interna, Especialista en Hematología Clínica. Hospital Universitario San Vicente Fundación, Universidad de Antioquia. Medellín, Colombia.

Conflicto de interés: los autores declaran que no tienen conflicto de interés.

Medicina \& Laboratorio 2021;25:695-707. https://doi.org/10.36384/01232576.524.

Recibido el 14 de agosto de 2021; aceptado el 10 de septiembre de 2021. Editora Médica Colombiana S.A., 2021 ${ }^{\circ}$.
} 
Palabras clave: virus de la inmunodeficiencia humana, síndrome de la inmunodeficiencia adquirida, infecciones por VIH, linfoma relacionado con SIDA, linfoma no Hodgkin, linfoma de Hodgkin.

Abstract. In Colombia, human immunodeficiency virus (HIV) infection is a growing epidemic. Given the improvement in overall survival with highly effective antiretroviral therapy, cancer has been displacing infectious complications in this population. Non-Hodgkin's lymphoma is the most common AIDS defining malignancy after Kaposi's sarcoma. Diffuse large B-cell lymphoma and Burkitt's lymphoma are the most common variants; meanwhile, the risk of developing classical Hodgkin lymphoma is 5 to 20-fold higher compared to HIV-negative individuals. Making an early diagnosis is a challenge, due to opportunistic infections and the atypical presentation of the disease in this group of patients. Treatment of the disease is also a challenge given the comorbidities and patient condition at diagnosis; however, in conjunction with combined chemotherapy regimens and the concomitant use of antiretroviral therapy, the outcome of patients with AIDS-related lymphomas has significantly improved, currently approaching that of seronegative patients. The aim of this review article is to describe the main characteristics of the disease, in such a way that it allows health workers to have more elements for a comprehensive approach in patients with AIDS-related lymphomas in our country.

Keywords: human immunodeficiency virus, acquired immunodeficiency syndrome, HIV infections, AIDS-related lymphoma, non-Hodgkin lymphoma, Hodgkin lymphoma.

\section{Introducción}

La infección por el virus de la inmunodeficiencia humana (VIH) y el síndrome de inmunodeficiencia adquirida (SIDA) son una problemática global con diversas y profundas implicaciones a nivel social e individual [1]. Con la mejora de la terapia antirretroviral (ART), se han ido desplazando las complicaciones infecciosas y ganan importancia otros problemas, como el riesgo de aparición de neoplasias malignas en estos pacientes [2]. Por su complejidad y frecuencia, las enfermedades linfoproliferativas en pacientes con VIH deben recibir especial atención. Usualmente, son condiciones agresivas que demandan terapia costosa de alta complejidad, con requerimiento de múltiples soportes e importante riesgo de complicaciones. Sin embargo, tienen la posibilidad de alcanzar buenos desenlaces, más allá de la generalizada imagen nihilista de este contexto clínico [3].

\section{Epidemiología general}

La infección por VIH afecta actualmente a casi 40 millones de personas en el mundo, quienes en su mayoría viven en países de bajos y medianos ingresos; siendo la principal causa de muerte de origen infeccioso, con casi 1,8 millones de fallecimientos anualmente [1]. Desde el inicio de la epidemia, se reconoció a las neoplasias malignas como otro problema importante para estos pacientes. Los estudios epide- 
miológicos concuerdan en reportar que predominan las neoplasias malignas definitorias de SIDA (AIDS Defining Cancer, $A D C$ ) que incluyen el sarcoma de Kaposi, el linfoma no Hodgkin y el carcinoma invasor de cérvix. Pero, con la reconstitución inmune por la ART y la consecuente prolongación de la supervivencia, el grupo de entidades conocidas como no definitorias de SIDA (NADC) como el linfoma de Hodgkin, carcinomas escamosos en otras localizaciones, neoplasias digestivas y el hepatocarcinoma, han ido aumentando progresivamente en prevalencia $[2,4]$ (tabla 1).

Se debe resaltar que las neoplasias hematológicas malignas son el subgrupo más importante asociado al $\mathrm{VIH}$. No solo son muy frecuentes en estos pacientes, sino que desde el punto de vista biológico son heterogéneas, más agresivas y demandan tratamientos más complejos en comparación con los comúnmente usados en casos de sarcoma de Kaposi y carcinoma invasor de cérvix. Así, los linfomas B lideran la lista de prevalencia, mientras que la leucemia y el mieloma múltiple no parecen aumentar en pacientes con $\mathrm{VIH}$ [5].

\section{Epidemiología con enfoque regional}

Un reciente estudio ecológico sobre la situación de la epidemia de $\mathrm{VIH}$ en Colombia [6], señala que de 2010 a 2018 se registró un incremento del $7 \%$ en la incidencia de la infección. Respecto a la asociación de ambas condiciones, el trabajo de Fink y colaboradores es el más grande realizado con población latinoamericana; agrupó registros de Argentina, Brasil, Chile, Haití, Honduras, México y Perú, e incluyó 428 pacientes con VIH y cáncer. De estos, el $82 \%$ presentó entidad definitoria de SIDA, siendo la principal
Tabla 1. Neoplasias malignas asociadas al VIH de acuerdo con su condición de marcadoras de inmunodeficiencia

\section{Definitorias de SIDA (grupo más frecuente)}

- Linfomas no Hodgkin (linfoproliferativas)

- Linfoma B difuso de células grandes

- Linfoma B de alto grado con o sin translocaciones recurrentes

- Linfoma de Burkitt

- Linfoma primario del sistema nervioso central

- Linfoma plasmablástico

- Linfoma primario de cavidades

- Enfermedad de Castleman multicéntrica

- Sarcoma de Kaposi (endotelial)

- Carcinoma invasor de cérvix (epitelial)

\section{No definitorias de SIDA (grupo en aumento)}

- Linfoma de Hodgkin (linfoproliferativa)

- Cáncer de piel no especificado

- Carcinoma no invasor de cérvix

- Carcinoma de vagina, vulva y canal anal

- Carcinoma de pene

- Hepatocarcinoma

- Carcinoma orofaríngeo

- Adenocarcinoma de estómago

- Carcinoma colorrectal

el sarcoma de Kaposi, seguido por el linfoma no Hodgkin (principalmente de alto grado), de los cuales un $74 \%$ recibió tratamiento con quimioterapia no especificada (con o sin radioterapia) [7]. Por otro lado, el trabajo más grande disponible a la fecha sobre la epidemiología del linfoma no Hodgkin asociado a $\mathrm{VIH}$, fue adelantado por el Grupo de Trabajo en Cáncer Definitorio de SIDA (The AIDS-Defining Cancer Project Working Group), que recogió datos de diferentes grupos de registro alrededor del mundo. Se incluyeron para seguimiento 210.898 pacientes, registrando 1.552 casos de linfoma no Hodgkin, durante un periodo de observación promedio de 4 años; concordando con otros estudios, la ma- 
yoría de los casos fueron hombres, en la quinta década de la vida, y con una incidencia de linfoma más alta en pacientes mayores con niveles más bajos de linfocitos CD4 [8].

En 2017, un trabajo del Instituto $\mathrm{Na}$ cional de Cancerología con sede en Bogotá, presentó datos de 139 pacientes con VIH y cáncer, la mayoría (casi el 70\%) con una entidad definitoria de SIDA; de estos, un poco más de la mitad tenían linfoma no Hodgkin no especificado (figura 1). Además, reportaron que la mitad de los diagnósticos de cáncer ocurrieron después de detectar la infección por $\mathrm{VIH}$, un $22,3 \%$ en simultáneo y un $16,6 \%$ antes de conocer la infección por el virus [9]. Por su parte, en el estudio de Uribe y colaboradores, que usó como fuente de los datos la Cuenta de Alto Costo (CAC) nacional, con la cual cruzaron los diagnósticos entre 1998 y 2015 (17 años), encontraron sorpresivamente un número de tan solo 238 casos de VIH y cáncer, lo cual sugiere de entra- da un posible subregistro de la coexistencia de estos eventos de interés en Colombia. Igual que otros trabajos latinoamericanos, el principal tipo de cáncer en seropositivos es el sarcoma de Kaposi, seguido del linfoma no Hodgkin. Esta publicación también presentó la distribución de los casos por departamentos (figura 2), advirtiendo que el $50 \%$ se agruparon con una distribución casi igual entre dos departamentos: Cundinamarca (28\%) y Antioquia (25,62\%) [10].

\section{Linfomagénesis en el paciente con VIH}

El entendimiento de los mecanismos moleculares de la infección viral que participan en el desarrollo de linfomas y otras neoplasias, es fundamental en el planteamiento de estrategias para la prevención, diagnóstico y tratamiento de estas patologías [11]. Es conocido que el sistema inmune tiene una clara función antineoplásica determinada

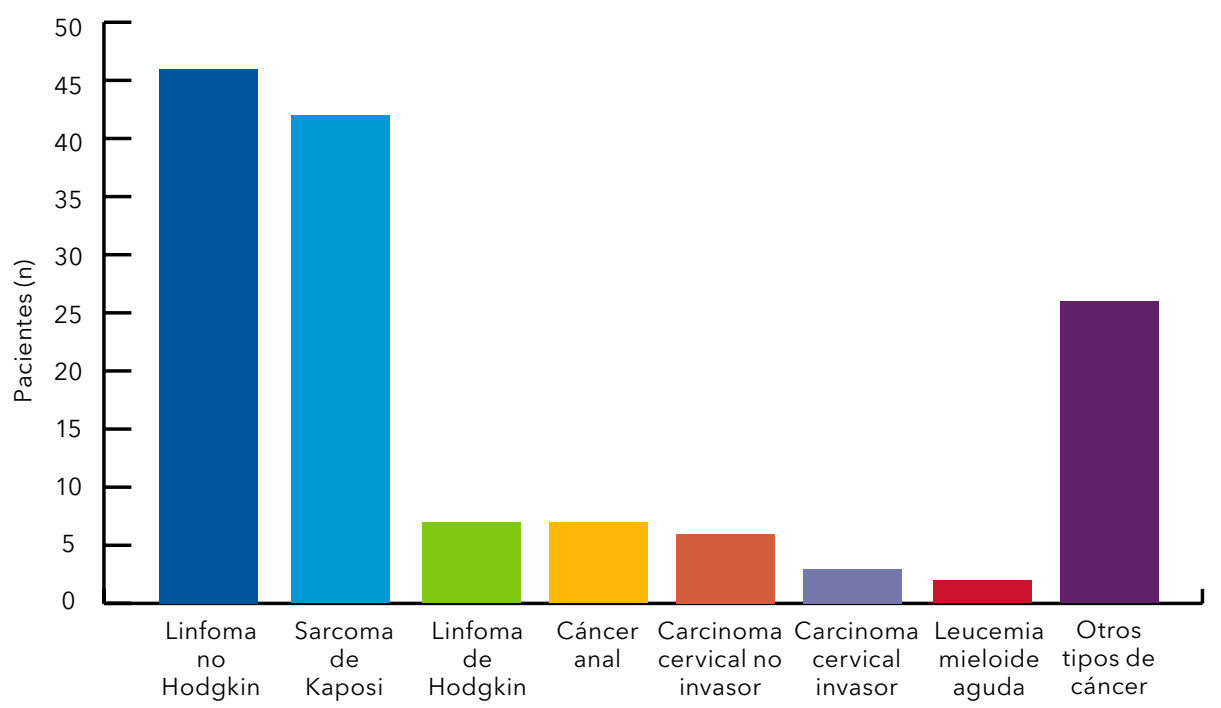

Figura 1. Distribución de neoplasias asociadas a VIH en el registro del Instituto Nacional de Cancerología en Colombia de 2007 a 2014. Tomado y adaptado [9]. 


\section{Casos $(n=238)$}

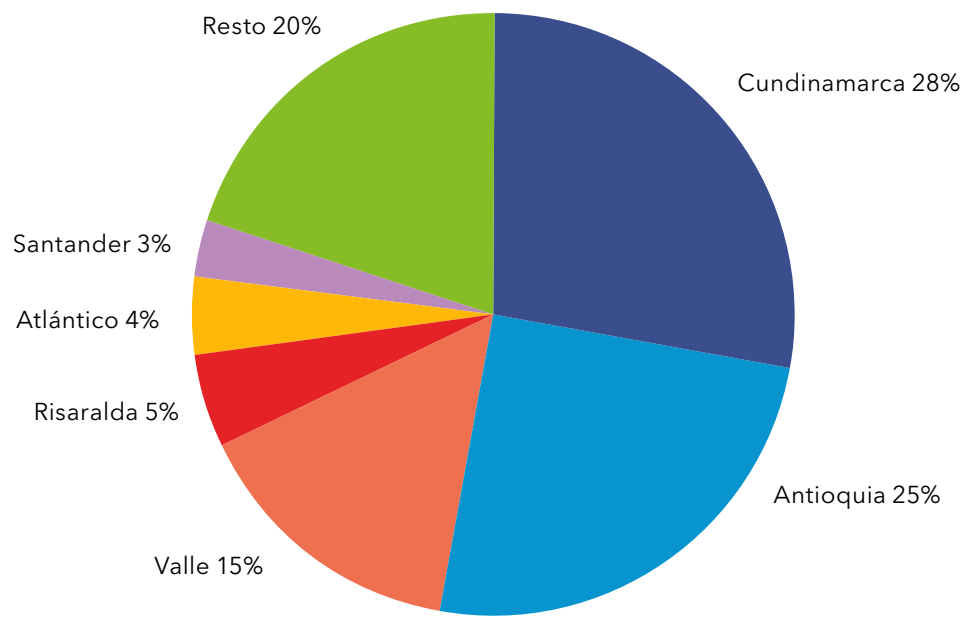

Figura 2. Distribución de casos de VIH y cáncer según la Cuenta de Alto Costo durante el periodo 1998 a 2015. Tomado y adaptado [10].

principalmente por el componente celular, es decir, controlada por linfocitos [12]. De esta manera, no es de extrañar que, en el contexto de inmunosupresión por $\mathrm{VIH}$, los procesos proliferativos se escapen de la vigilancia inmune y puedan evolucionar a francas neoplasias malignas. Además de la inmunosupresión, se reconocen otros factores asociados con la mayor incidencia de linfomas en los pacientes positivos para $\mathrm{VIH}$ : alta prevalencia de coinfección con oncovirus de transmisión sexual y parenteral, mayor frecuencia de consumo de alcohol y cigarrillo, mayor incidencia de procesos inflamatorios crónicos (estimulación antigénica), y aumento de la franja de supervivientes con edades más avanzadas [2,13]. Dichos factores explican parte de la susceptibilidad aumentada en este grupo de pacientes, y algunas diferencias epidemiológicas que muestran un peor panorama del fenómeno cáncer y VIH en los países en vía de desarroIlo, donde pueden coexistir con más frecuencia varias de las características de riesgo. Por ejemplo, Wiggill y colaboradores señalaron un incremento de la prevalencia de linfomas en una cohorte africana, lo que se asoció con un aumento concurrente de la seropositividad por $\mathrm{VIH}$, llegando a coexistir en un $62 \%$ de los casos de linfoma y hasta en el $90 \%$ en el subgrupo de linfomas agresivos de alto grado, respaldando que en la práctica hay una fuerte relación entre ambas condiciones [14].

Recientemente, han ganado relevancia datos a favor de un efecto linfomagénico directo del VIH sobre las células linfoides. Algunas proteínas virales podrían interferir en vías de señalización tumoral [15] o inducir cambios de componentes del microambiente que favorecen el desarrollo de las células malignas. Esta interrelación entre el estroma, estructuras vasculares y sistema inmune son tan importantes como los cambios inducidos directamente en las células tumorales [16]. También, se ha propuesto la 
inducción de neoangiogénesis como un factor fundamental en el desarrollo e invasión por linfoma en el contexto de infección por $\mathrm{VIH}$ [11], al igual que otros componentes como la liberación anormal de citoquinas, y la coinfección disregulada con otros virus como el de Epstein-Barr y el HHV-8, entre otros.

Por otro lado, es claro que las enfermedades linfoproliferativas asociadas al $\mathrm{VIH}$ son biológicamente más agresivas. Pero, paradójicamente, los estudios de secuenciación que las comparan con linfomas en pacientes inmunocompetentes muestran un contexto mutagénico más discreto, lo que lleva a suponer que los linfomas asociados a VIH requieren menos alteraciones genéticas para superar el control inmune y desarrollarse [17]. No obstante, aún hay muchas oportunidades de investigación para ampliar el conocimiento en la definición molecular de la neoplasia en este grupo específico de pacientes.

\section{Manifestaciones clínicas y diagnóstico}

Los linfomas asociados al VIH pueden presentarse en cualquier estadio de la enfermedad, pero por lo general aparecen con recuentos de linfocitos T CD4 inferiores o iguales a 200 células $/ \mathrm{mm}^{3}$. El cuadro clínico es bastante heterogéneo, especialmente considerando que los pacientes con mayor compromiso inmune pueden presentar procesos infecciosos oportunistas concomitantes y otras complicaciones, como desnutrición o compromiso orgánico relacionado con la infección retroviral. Entonces, se sugiere al clínico mantener una actitud de alta sospecha diagnóstica procurando apoyarse de forma racional de varios recursos, incluyendo la confirmación histopatológica, con el fin de mejorar la oportunidad y el rendimien- to diagnóstico en estos casos. Siendo pragmáticos, se debe considerar la posibilidad de linfoma en todo paciente con $\mathrm{VIH}$, y de $\mathrm{VIH}$ en todo paciente con diagnóstico nuevo de linfoma, con el fin de enfocar el manejo y estudios según el contexto. Así mismo, son sospechosos de linfoma aquellos pacientes seropositivos con adenopatías palpables o cavitarias múltiples, y aquellos que presentan síntomas constitucionales marcados o lesiones focales sospechosas de neoplasia, especialmente si están localizadas en el sistema nervioso central o que se asocien con adenomegalia o hepatoesplenomegalia.

Lamentablemente, el diagnóstico es muy complejo por la gran cantidad de diferenciales con clínica indistinguible a la del linfoma. Así, hay varias condiciones infecciosas que incluso pueden inducir procesos histopatológicos reactivos, como el síndrome hemofagocítico [18] o la enfermedad de Castleman multicéntrica [19], similares desde toda perspectiva a un trastorno linfoproliferativo. Por otro lado, pueden presentarse formas atípicas de linfoma como lesiones primarias extranodales de localización inusual, que llevan a considerar otros tipos de neoplasia o procesos benignos, retrasando la confirmación de malignidad de origen linfoide. La tuberculosis (TB), por ejemplo, que está presente con relativa frecuencia en los pacientes infectados con $\mathrm{VIH}$, produce manifestaciones extrapulmonares que incluyen adenopatías y síntomas constitucionales (fiebre, sudoración nocturna y pérdida de peso), imitando al linfoma [14]. Lo contrario también puede ocurrir, en particular en áreas endémicas para tuberculosis, en donde los clínicos pueden atribuir los síntomas a la infección micobacteriana e iniciar tratamiento empírico para $\mathrm{TB}$, sin tenerse en cuenta la posibilidad del linfoma como diagnóstico en el paciente con $\mathrm{VIH}$ [20]. Y en el 
último escenario, que no es infrecuente, se debe tener en cuenta la coexistencia de una neoplasia linfoproliferativa con uno o varios oportunistas. En esta situación es indispensable contar con diferentes muestras de secreción y tejido para un cuidadoso análisis microbiológico y patológico, ojalá con disponibilidad de pruebas avanzadas de histoquímica, genética y biología molecular, para no pasar por alto ninguna de las posibilidades $[21,22]$.

Hay que resaltar que el diagnóstico de los linfomas requiere estudios de biopsia de tejido para histopatología e inmunohistoquímica, que deben ser leídos por un patólogo con experiencia en hematopatología [23,24], porque la lista de linfomas en $\mathrm{VIH}$ es extensa, y cada uno de ellos tiene una presentación característica, que en ocasiones pueden distorsionarse por la inmunosupresión [16]. Por lo tanto, es necesario la adquisición de claves diagnósticas que permitan al médico contar con la mayor cantidad de recursos posibles para el direccionamiento preciso y oportuno de estos pacientes (tabla 2) [2,25].

\section{Tratamiento}

En la práctica, la coexistencia del VIH/ SIDA y la enfermedad linfoproliferativa significa un reto para los médicos tratantes, quienes se enfrentan a múltiples factores dinámicos en un mismo paciente: inmunosupresión, comportamiento biológico agresivo (enfermedad avanzada y extranodal), infección oportunista, profilaxis, citopenias, interacciones farmacológicas, toxicidad, desnutrición, otros soportes, etc. [26]. Por fortuna, la evidencia y experiencia disponibles han ido aclarando poco a poco la hoja de ruta con este tipo de pacientes, a quienes en la actualidad se les puede ofrecer muy buenas expectativas con intención curativa por medio de antirretrovirales y quimioterapia intensa, que deben ser manejados teniendo en cuenta los riesgos asociados en esta población de pacientes $[23,24,27]$. Sobra decir que estos logros solo serán alcanzados si los médicos se apoyan en un grupo interdisciplinario que pueda comunicarse oportunamente según lo vaya requiriendo el caso.

Son variadas las entidades neoplásicas que afectan al paciente con $\mathrm{VIH}$, por lo cual no es posible definir un tratamiento único para todas. No obstante, algunos datos derivados de estudios clínicos permiten hacer ciertas recomendaciones más o menos generales. Primero, es prioritario iniciar y ajustar la ART para favorecer la reconstitución inmune, que a su vez, mejorará la respuesta a la terapia antineoplásica. Segundo, la mayoría de los pacientes con linfomas de células B se benefician del uso de anticuerpos monoclonales antiCD20 dentro de los esquemas de tratamiento de quimioterapia, con ventajas en su supervivencia [28]. Al respecto, aún hay discusiones alrededor del riesgo de empeorar la linfopenia en un paciente inmunodeficiente, así que algunos autores difieren el inicio de rituximab hasta tener recuentos de CD4 de al menos 50 células $/ \mathrm{mm}^{3}$. Tercero, la quimioterapia en regímenes intensos es posible y segura, además que parece superior a esquemas menos densos. En este punto, la comparación más importante ha sido entre la combinación de ciclofosfamida, doxorrubicina, vincristina y prednisona (R-CHOP), contra el esquema equivalente pero con adición de etopósido y de administración infusional ajustada por toxicidad, conocido como R-DA-EPOCH. Hasta el momento, la evidencia, al menos en el escenario de linfomas de alto y muy 
alto grado que son los más frecuentes en pacientes con $\mathrm{VIH}$, favorece el uso de R-DA-EPOCH $[26,29]$. Cuarto, hay consenso en que los pacientes con $\mathrm{VIH}$ podrían tener un riesgo aumentado de presentar compromiso secundario en sistema nervioso central, por lo cual se recomienda evaluar esta opción con estudio de enfermedad oculta, analizando el líquido cefalorraquídeo en el diagnóstico con citología y citometría de flujo, además de considerar neuroimagen preferiblemente con resonancia cerebral contrastada, si hay sínto-

Tabla 2. Claves diagnósticas en tumores linfoproliferativos asociados al VIH. Tomado y adaptado [2]

\begin{tabular}{|c|c|}
\hline $\begin{array}{l}\text { Enfermedades linfoproliferativas } \\
\text { asociadas al VIH }\end{array}$ & Clave diagnóstica \\
\hline Linfoma B difuso de células grandes & $\begin{array}{l}\text { - Virus de Epstein-Barr (-) } \\
\text { - Es el más común en pacientes VIH (+) } \\
\text { - Afectación ganglionar o extraganglionar } \\
\text { - Afectación del SNC } \\
\text { - Aumento de LDH } \\
\text { - Posible presencia de translocación C-MYC } \\
\text { - CD20 (+) } \\
\text { - BCL6 (+) } \\
\text { - Coexpresión de MUM1/IRF4 y BCL6 }\end{array}$ \\
\hline $\begin{array}{l}\text { Linfoma B de alto grado con o sin } \\
\text { translocaciones recurrentes }\end{array}$ & $\begin{array}{l}\text { - } 70 \% \text { a } 100 \% \text { en estadio IV } \\
\text { - Localización mayoritariamente extranodal: médula } \\
\text { ósea y SNC } \\
\text { - LDH elevada } \\
\text { - Translocación de BCL6 y BCL2 } \\
\text { - Translocación de C-MYC en el } 50 \%\end{array}$ \\
\hline Linfoma de Burkitt & $\begin{array}{l}\text { - Virus de Epstein-Barr (+) } \\
\text { - Aumento de LDH y ácido úrico } \\
\text { - Presencia de translocación de C-MYC } \\
\text { - Morfología inmunoblástica característica } \\
\text { - CD20 (+) } \\
\text { - CD10 (+) }\end{array}$ \\
\hline $\begin{array}{l}\text { Linfoma primario del sistema nervioso } \\
\text { central }\end{array}$ & $\begin{array}{l}\text { - Virus de Epstein-Barr (+) } \\
\text { - Masas en SNC realzadas en anillo } \\
\text { - Comunes las infecciones en SNC } \\
\text { - CD4 }(+)<100 \text { células } / \mathrm{mm}^{3}\end{array}$ \\
\hline Linfoma plasmablástico & $\begin{array}{l}\text { - Virus de Epstein-Barr (+) } \\
\text { - Lesiones en cavidad oral y tracto gastrointestinal } \\
\text { - Inmunoblastos y diferenciación plasmocítica } \\
\text { - Presencia de translocaciones de C-MYC } \\
\text { - CD79a (+) } \\
\text { - CD20 (-) } \\
\text { - BCL2 y BCL6 (+) }\end{array}$ \\
\hline
\end{tabular}




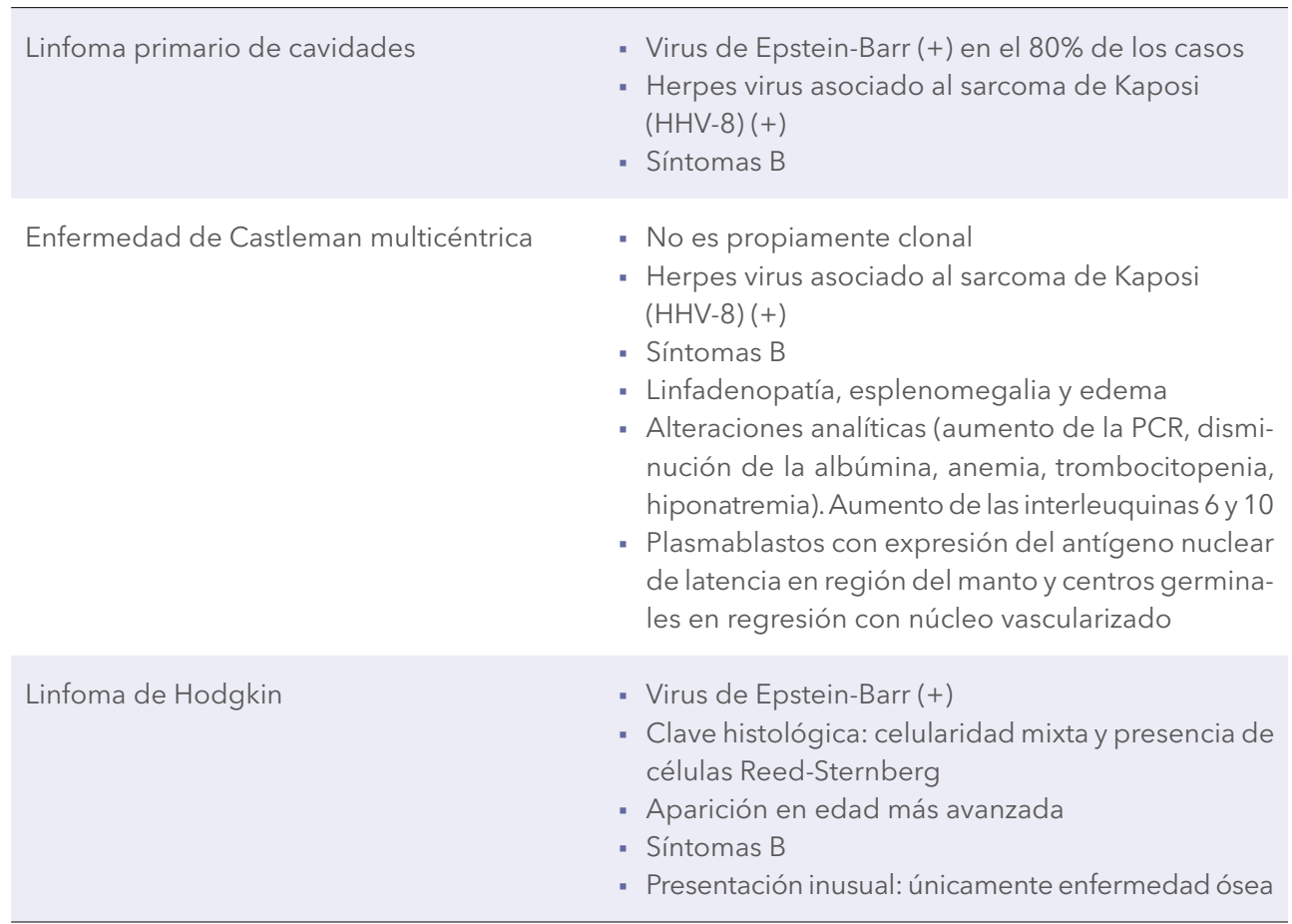

SNC: sistema nervioso central; LDH: deshidrogenasa láctica; HHV-8: virus herpes humano 8.

mas sugestivos [30]. De confirmarse la enfermedad en el sistema nervioso, deberá adaptarse el esquema de terapia sistémica para incluir el neuroeje. De lo contrario, se acepta el uso de profilaxis con quimioterapia intratecal en estos pacientes. Si bien no hay una contraindicación para seleccionar altas dosis sistémicas de metotrexato como esquema de profilaxis, se considera que el riesgo de interacción y toxicidad en este subgrupo de pacientes favorece, al menos desde el punto de vista práctico, a la terapia intratecal, mientras se logra mejor evidencia que defina la superioridad entre un esquema u otro.

Por último, y no menos importante, para poder garantizar el éxito de las estrategias de tratamiento descritas anteriormente, es fundamental tener en cuenta otras medidas de soporte general [31], por ejemplo, usar siempre que sea posible estimulantes de colo- nias de granulocitos en los esquemas de quimioterapia, y dar cubrimiento con profilaxis antimicrobiana, incluyendo no solo a los gérmenes oportunistas conocidos para los pacientes con SIDA, sino los que ocurren en los pacientes sometidos a quimioterapia. Adicionalmente, dar soporte transfusional en caso de requerirse, señalando que la infección por VIH per se no es indicación de productos irradiados, e iniciar rehabilitación integral con el apoyo de nutrición, fisioterapia y psicología, según se requiera.

Sale de los alcances de esta revisión, describir la terapia específica de cada uno de los tipos de linfomas que pueden ocurrir en el paciente con VIH. Sin embargo, sugerimos al lector consultar las guías de la NCCN (National Comprehensive Cancer Network) y ESMO (European Society for Medical Oncology) periódicamente, porque se prevén 
futuras modificaciones de las opciones terapéuticas disponibles conforme se van presentando los resultados de varios estudios que exploran el riesgo y beneficio de la introducción de nuevos fármacos en esta población, como es el caso de brentuximab en pacientes con linfoma de Hodgkin y VIH [32], o de bortezomib y daratumumab en aquellos con linfoma plasmablástico y $\mathrm{VIH}$ [33].

El trasplante autólogo de precursores hematopoyéticos es posible y está recomendado dentro de los esquemas de tratamiento de primera y segunda línea de algunos de los tipos de linfoma en pacientes con VIH [34]. Por su parte, el trasplante alogénico y haploidéntico tienen menos evidencia de respaldo, pero existe gran interés en su investigación, especialmente porque se han reportado casos exitosos incluyendo aquellos que derivaron en la "curación" (remisión a largo plazo sin tratamiento) del VIH como un logro secundario (pacientes en Berlín, Londres y Düsseldorf) [35].

Lamentablemente, a pesar de los logros persiste una percepción nihilista de los médicos hacia los pacientes con VIH y cáncer, lo que aún promueve la modificación de las dosis e incluso desestimar la terapia intensiva por temor injustificado a complicaciones derivadas del tratamiento, lo que limita la posibilidad de curación (u otros desenlaces) en pacientes elegibles para este enfoque, siempre de la mano de terapia de soporte integral $[3,36]$.

\section{Pronóstico}

Múltiples factores relacionados con el paciente o su patología son contribuyentes al pronóstico, y por lo tanto, a la supervivencia de este, siendo los niveles de CD4 el factor predictor más significa-
Tabla 3. Determinantes de la enfermedad por $\mathrm{VIH}$ y linfoma. Tomado y adaptado [36]

\section{Asociados con el linfoma}

- Histología

- Estadio

- Tamaño tumoral

- Localización

- IPI (Índice Pronóstico Internacional)

\section{Asociados al VIH}

- Perfil inmunovirológico (CD4 y carga viral)

- Infecciones oportunistas

- Terapia antirretroviral

\section{Asociados al paciente}

- Edad

- ECOG (Eastern Cooperative Oncology Group)

- Comorbilidades

- Preferencias del paciente

\section{Asociados al tratante}

- Experiencia en el tratamiento de linfoma

- Experiencia en el tratamiento de VIH

tivo en estos individuos (tabla 3). Según un estudio llevado a cabo en 100 pacientes en el Hospital Provincial de Enfermedades Infecciosas de Henan en China, factores como una edad menor de 45 años, niveles de CD4 $<100$ células $/ \mathrm{mm}^{3}$, un Índice Pronóstico Internacional (IPI) alto y un nivel elevado de LDH, son los predictores de peor pronóstico para un paciente positivo para VIH con enfermedad linfoproliferativa concomitante [37].

Por fortuna, el desarrollo de la ART combinada ha contribuido a mejorar el pronóstico de la enfermedad en los diferentes subtipos de linfoma, hallándose una diferencia sustancial en la supervivencia en la era pre-ART y en la actual (tabla 4) [38]. En lo que respecta al linfoma de Hodgkin, según un estudio llevado a cabo por 


\begin{tabular}{lcc}
\hline Tabla 4. Supervivencia general de los subtipos de linfoma asociados al VIH. Tomado y adaptado [38] \\
\hline Tipos de linfoma & Era pre-ART (\%) & Era actual ART (\%) \\
\hline Linfoma de Burkitt & 10 a 40 & 70 a 80 \\
\hline Linfoma B difuso de células grandes & 40 & 70 a 80 \\
\hline Linfoma de Hodgkin & 55 & 80 a 90 \\
\hline Linfoma plasmablástico & 6 & 75 \\
\hline Linfoma primario del SNC & 20 & 60 \\
\hline Linfoma primario de cavidades & 33 & 40 \\
\hline
\end{tabular}

ART: terapia antirretroviral; SNC: sistema nervioso central.

el grupo español GESIDA, al comparar un grupo de 83 pacientes tratados y no tratados con esta terapia, se encontró que los pacientes que recibían ART tenían un perfil de remisión y supervivencia mayor que los que no tuvieron este manejo médico (91\% frente a un 70\%) [39].

\section{Conclusión}

Más allá de reconocer el claro aumento de la incidencia de malignidad en esta población, se debe comprender que la complejidad del problema radica en las características únicas de estos pacientes, que requieren de una aproximación juiciosa que no descuide ninguna de las determinantes que participan en la coexistencia de ambas entidades: cáncer y VIH/SIDA. También se deben aprovechar muchas oportunidades de investigación, especialmente en los países en vía de desarrollo aún subrepresentados en la literatura, y donde todavía se presentan condiciones paradójicas que permiten ver toda la gama del espectro epidemiológico, con pacientes seropositivos con limitación psicosocial grave, que los mantiene en la era pre-ART, y otros por el contrario que acceden fácilmente a la terapia ART logrando completo control de la enfermedad.

\section{Agradecimientos}

Agradecemos a la Doctora Vanessa Santiago Pacheco (especialista en Hematopatología) por su pertinente revisión y recomendaciones.

\section{Referencias}

1. United Nations. UNAIDS: Joint United Nations Programme on HIV/AIDS. New York, Estados Unidos: United Nations; 2015. Acceso 16 de julio de 2021. Disponible en https://www.un.org/ youthenvoy/2013/08/unaids-joint-united-nations-programme-on-hivaids/.

2. Yarchoan R, Uldrick TS. HIV-associated cancers and related diseases. N Engl J Med 2018;378:1029-1041. https://doi.org/10.1056/ NEJMra1615896.

3. Noy A. HIV and lymphoma: from oncological futility to treatment. Lancet HIV 2020;7:e598-e600. https://doi. org/10.1016/s2352-3018(20)30227-7.

4. Morlat P, Roussillon C, Henard S, Salmon D, Bonnet F, Cacoub P, et al. Causes of death among HIV-infected patients in France in 2010 (national survey): trends since 2000. Aids 2014;28:1181-1191. https://doi.org/10.1097/ qad.0000000000000222.

5. Kimani SM, Painschab MS, Horner MJ, Muchengeti M, Fedoriw Y, Shiels MS, et al. Epidemiology of haematological malignancies in people living with HIV. Lancet HIV 
2020;7:e641-e651. https://doi.org/10.1016/ s2352-3018(20)30118-1.

6. Montana JF, Ferreira G, Cunha CLF, de Queiroz AAR, Fernandes WAA, Polaro SHI, et al. The HIV epidemic in Colombia: spatial and temporal trends analysis. BMC Public Health 2021;21:178. https://doi.org/10.1186/s12889021-10196-y.

7. Fink VI, Shepherd BE, Cesar C, Krolewiecki A, Wehbe F, Cortés CP, et al. Cancer in HIVinfected persons from the Caribbean, Central and South America. J Acquir Immune Defic Syndr 2011;56:467-473. https://doi.org/10.1097/ qai.0b013e31820bb1c3.

8. AIDS-defining Cancer Project Working Group of leDEA, COHERE in EuroCoord. Non-Hodgkin lymphoma risk in adults living with HIV across five continents. Aids 2018;32:2777-2786. https:// doi.org/10.1097/qad.0000000000002003.

9. Álvarez-Guevara $\mathrm{D}$, Cuervo-Maldonado $\mathrm{S}$, Sánchez R, Gómez-Rincón J, Ramírez N. Prevalence of defining malignancies in adult patients with HIV/AIDS in the National Cancer Institute of Colombia. 2007-2014. Rev Fac Med 2017;65:397-402.

10. Uribe-Parra D, Pulido D, Lopes G, Martinez $P$, Acuña L, Valencia O, et al. Cancer incidence in patients diagnosed with acquired immunodeficiency syndrome (AIDS) in Colombia. J AIDS Clin Res 2017;08. https://doi.org/10.4172/21556113.1000692.

11. Carbone A, Vaccher E, Gloghini A, Pantanowitz $\mathbf{L}$, Abayomi A, de Paoli $\mathbf{P}$, et al. Diagnosis and management of lymphomas and other cancers in HIV-infected patients. Nat Rev Clin Oncol 2014;11:223-238. https://doi. org/10.1038/nrclinonc.2014.31.

12. Candeias $\mathbf{S M}$, Gaipl US. The immune system in cancer prevention, development and therapy. Anticancer Agents Med Chem 2016;16:101 107. https://doi.org/10.2174/18715206156661 50824153523.

13. Silverberg MJ, Chao C, Leyden WA, Xu L, Tang B, Horberg MA, et al. HIV infection and the risk of cancers with and without a known infectious cause. Aids 2009;23:2337-2345. https://doi. org/10.1097/QAD.0b013e3283319184.

14. Wiggill TM, Mantina $H$, Willem $P$, Perner $Y$, Stevens WS. Changing pattern of lymphoma subgroups at a tertiary academic complex in a high-prevalence HIV setting: a South African perspective. J Acquir Immune Defic Syndr 2011;56:460-466. https://doi.org/10.1097/ QAl.0b013e31820bb06a.

15. Martorelli D, Muraro E, Mastorci K, Dal Col J, Faè DA, Furlan $\mathbf{C}$, et al. A natural HIV p17 protein variant up-regulates the LMP-1 EBV oncoprotein and promotes the growth of EBV-infected B-lymphocytes: implications for EBV-driven lymphomagenesis in the HIV setting. Int J Cancer 2015;137:1374-1385. https://doi.org/10.1002/ ijc. 29494

16. Pantanowitz L, Carbone A, Dolcetti R. Microenvironment and HIV-related lymphomagenesis. Semin Cancer Biol 2015;34:52-57. https:// doi.org/10.1016/j.semcancer.2015.06.002.

17. Zhang J, Grubor V, Love CL, Banerjee A, Richards KL, Miezcowski P, et al. The genetic landscape of immune-competent and HIV lymphoma. Infect Agent Cancer 2012;7:01. https://doi.org/10.1186/1750-9378-7-S1-O1.

18. Castelli AA, Rosenthal DG, Bender Ignacio R, Chu HY. Hemophagocytic lymphohistiocytosis secondary to human immunodeficiency virusassociated histoplasmosis. Open Forum Infect Dis 2015;2:ofv140. https://doi.org/10.1093/ ofid/ofv140.

19. Reddy D, Mitsuyasu R. HIV-associated multicentric Castleman disease. Curr Opin Oncol 2011;23:475-481. https://doi.org/10.1097/ cCO.0b013e328349c233.

20. Antel $K$, Levetan $C$, Mohamed $Z$, Louw VJ, Oosthuizen J, Maartens G, et al. The determinants and impact of diagnostic delay in lymphoma in a TB and HIV endemic setting. BMC Cancer 2019;19:384. https://doi.org/10.1186/ s12885-019-5586-4

21. Cortez MV, Oliveira $C M$, Monte RL, Araújo JR, Braga BB, Reis DZ, et al. HIV-associated tuberculous lymphadenitis: the importance of polymerase chain reaction (PCR) as a complementary tool for the diagnosis of tuberculosis - a study of 104 patients. An Bras Dermatol 2011;86:925-931. https:// doi.org/10.1590/s0365-05962011000500010.

22. Shao L, Jiang L, Wu S, Yu L, Wang L, Huang X. Simultaneous occurrence of invasive pulmonary aspergillosis and diffuse large B-cell lymphoma: case report and literature review. BMC 
Cancer 2020;20:15. https://doi.org/10.1186/ s12885-019-6471-x.

23. Kaplan LD. HIV-associated lymphoma. Best Pract Res Clin Haematol 2012;25:101-117. https://doi.org/10.1016/j.beha.2012.01.001.

24. Brunnberg $U$, Hentrich $M$, Hoffmann $C$, Wolf T, Hübel K. HIV-associated malignant lymphoma. Oncol Res Treat 2017;40:82-87. https://doi. org/10.1159/000456036.

25. International Agency for Research on Cancer (IARC). WHO Classification of Tumours of Haematopoietic and Lymphoid Tissues. 4th ed ed. Lyon: International Agency for Research on Cancer (IARC); 2017. p. 581

26. Barta $S K$, Xue $X$, Wang $D$, Tamari R, Lee JY, Mounier N, et al. Treatment factors affecting outcomes in HIV-associated non-Hodgkin lymphomas: a pooled analysis of 1546 patients. Blood 2013;122:3251-3262. https://doi. org/10.1182/blood-2013-04-498964.

27. Calkins KL, Chander G, Joshu CE, Visvanathan $\mathbf{K}$, Fojo AT, Lesko CR, et al. Immune status and associated mortality after cancer treatment among individuals with HIV in the antiretroviral therapy era. JAMA Oncol 2020;6:227-235. https://doi.org/10.1001/jamaoncol.2019.4648.

28. Sparano JA, Lee JY, Kaplan LD, Levine AM, Ramos JC, Ambinder RF, et al. Rituximab plus concurrent infusional EPOCH chemotherapy is highly effective in HIV-associated B-cell non-Hodgkin lymphoma. Blood 2010;115:3008-3016. https://doi.org/10.1182/ blood-2009-08-231613.

29. Barta SK, Lee JY, Kaplan LD, Noy A, Sparano JA. Pooled analysis of AIDS malignancy consortium trials evaluating rituximab plus $\mathrm{CHOP}$ or infusional EPOCH chemotherapy in HIV-associated non-Hodgkin lymphoma. Cancer 2012;118:39773983. https://doi.org/10.1002/cncr.26723.

30. Hegde U, Filie A, Little RF, Janik JE, Grant N, Steinberg SM, et al. High incidence of occult leptomeningeal disease detected by flow cytometry in newly diagnosed aggressive B-cell lymphomas at risk for central nervous system involvement: the role of flow cytometry versus cytology. Blood 2005;105:496-502. https://doi. org/10.1182/blood-2004-05-1982.
31. National Comprehensive Cancer Network (NCCN). NCCN Guidelines. B-cell lymphomas. Pensilvania, Estados Unidos: National Comprehensive Cancer Network; 2021. Acceso 05 de mayo de 2021. Disponible en https://www.nccn.org/guidelines/guidelinesdetail?category=1\&id=1480.

32. Rubinstein PG, Moore PC, Rudek MA, Henry DH, Ramos JC, Ratner L, et al. Brentuximab vedotin with AVD shows safety, in the absence of strong CYP3A4 inhibitors, in newly diagnosed HIV-associated Hodgkin lymphoma. Aids 2018;32:605-611. https://doi.org/10.1097/ qad.0000000000001729.

33. Castillo JJ, Reagan JL, Sikov WM, Winer ES. Bortezomib in combination with infusional dose-adjusted EPOCH for the treatment of plasmablastic lymphoma. $\mathrm{Br} \mathrm{J}$ Haematol 2015;169:352-355. https://doi.org/10.1111/ bjh.13300.

34. Alvarnas JC, Le Rademacher J, Wang Y, Little RF, Akpek G, Ayala E, et al. Autologous hematopoietic cell transplantation for HIV-related lymphoma: results of the BMT CTN 0803/AMC 071 trial. Blood 2016;128:1050-1058. https:// doi.org/10.1182/blood-2015-08-664706.

35. Hütter G, Nowak D, Mossner M, Ganepola S, Müssig A, Allers K, et al. Long-term control of HIV by CCR5 Delta32/Delta32 stem-cell transplantation. N Engl J Med 2009;360:692-698. https://doi.org/10.1056/NEJMoa0802905.

36. Meister A, Hentrich M, Wyen C, Hübel K. Malignant lymphoma in the HIV-positive patient. Eur J Haematol 2018;101:119-126. https://doi. org/10.1111/ejh.13082.

37. Wu D, Chen $C$, Zhang M, Li Z, Wang S, Shi J, et al. The clinical features and prognosis of 100 AIDS-related lymphoma cases. Sci Rep 2019;9:5381. https://doi.org/10.1038/ s41598-019-41869-9.

38. Noy A. Optimizing treatment of HIV-associated lymphoma.Blood 2019;134:1385-1394. https:// doi.org/10.1182/blood-2018-01-791400.

39. Jacobson CA, Abramson JS. HIV-associated Hodgkin's lymphoma: Prognosis and therapy in the era of cART.AdvHematol2012;2012:507257. https://doi.org/10.1155/2012/507257. 